\title{
Nicotianamine synthase overexpression positively modulates iron homeostasis-related genes in high iron rice
}

\author{
Meng Wang, Wilhelm Gruissem and Navreet K. Bhullar* \\ Plant Biotechnology, Department of Biology, Swiss Federal Institute of Technology, Zürich, Switzerland
}

\section{Edited by:}

Irene Murgia, Università degli Studi

di Milano, Italy

Reviewed by:

Beat Keller, University of Zurich, Switzerland

Rubén Rellán-Álvarez, Carnegie Institution for Science, USA

\section{*Correspondence:}

Navreet K. Bhullar, Plant

Biotechnology, Department of

Biology, Swiss Federal Institute

of Technology, Universitätstrasse 2, CH-8092 Zürich, Switzerland

e-mail: bhullarn@ethz.ch
Nearly one-third of the world population, mostly women and children, suffer from iron malnutrition and its consequences, such as anemia or impaired mental development. Biofortification of rice, which is a staple crop for nearly half of the world's population, can significantly contribute in alleviating iron deficiency. NFP rice (transgenic rice expressing nicotianamine synthase, ferritin and phytase genes) has a more than six-fold increase in iron content in polished rice grains, resulting from the synergistic action of nicotianamine synthase (NAS) and ferritin transgenes. We investigated iron homeostasis in NFP plants by analyzing the expression of 28 endogenous rice genes known to be involved in the homeostasis of iron and other metals, in iron-deficient and iron-sufficient conditions. RNA was collected from different tissues (roots, flag leaves, grains) and th three developmental stages during grain filling. NFP plants showed increased sensitivity to iron-deficiency conditions and changes in the expression of endogenous genes involved in nicotianamine (NA) metabolism, in comparison to their non-transgenic siblings (NTS). Elevated transcript levels were detected in NFP plants for several iron transporters. In contrast, expression of OsYSL2, which encodes a member of yellow stripe like protein family, and a transporter of the NA-Fe(II) complex was reduced in NFP plants under low iron conditions, indicating that expression of OsYSL2 is regulated by the endogenous iron status. Expression of the transgenes did not significantly affect overall iron homeostasis in NFP plants, which establishes the engineered push-pull mechanism as a suitable strategy to increase rice endosperm iron content.

Keywords: iron, homeostasis, NFP rice, biofortification, expression profiling

\section{INTRODUCTION}

Iron deficiency anemia (IDA) is the most severe degree of iron deficiency and a global problem that affects an estimated onethird of the world's population in both developing and developed countries. IDA has major consequences for human health as well as social and economic progress (WHO, 2013). Human IDA could be relieved by iron supplementation or food fortification. However, iron supplementation is difficult to achieve due to transportation and economic circumstances, especially in rural areas of developing countries. Iron fortification of food is also technically difficult and often results in unacceptable color and flavor of fortified products (Hurrell and Egli, 2010). In the recent years, bio-fortification has emerged as a possible solution to combat iron deficiency anemia through an economical and natural way.

Rice is the second largest produced cereal in the world and the most important grain with regard to human nutrition and caloric intake. It provides more than one fifth of the calories consumed worldwide. Around 3 billion people, mostly in Asia, depend on rice for $35-59 \%$ of their caloric intake. However, rice is a poor source of micronutrients, including iron. Most commercial rice varieties have only around $2 \mu \mathrm{g} / \mathrm{g}$ iron in the endosperm. Therefore, rice cannot provide daily iron needs of humans i.e., at least $8 \mathrm{mg} /$ day for males and $18 \mathrm{mg} /$ day for females, with pregnant women requirements rising up to $27 \mathrm{mg}$ /day (Institute of Medicine, 2013). Considering these facts, enrichment of rice endosperm with bioavailable iron has the potential to decrease iron malnutrition worldwide. However, iron biofortification of rice strongly relies on information on the genes that control iron homeostasis in plants.

Iron translocation and homeostasis in rice has been wellstudied. Several genes, most of which are transcriptionally regulated in response to iron availability, are known to coordinate iron uptake, translocation and storage in various tissues/compartments of the plant (Kobayashi and Nishizawa, 2012). However, the contribution of each type of transporter(s) and the precise iron flux still need to be clarified for each step involved in iron translocation. The transcription factors OSIDEF1 and OSIDEF2 regulate iron homeostasis-related genes in rice during Fe deficiency (Ogo et al., 2008; Kobayashi et al., 2009, 2010a). It has been suggested that OsIDEF1 senses the cellular iron status by binding directly to the metal ions (Kobayashi et al., 2012). To cope with Fe starvation, rice roots release phytosiderophores (PS), which are molecules of the mugineic acid (MAs) family that form strong hexadentate chelates with $\mathrm{Fe}$ (III)to solubilize and transport it to the plant (Walker and Connolly, 2008; Palmer and Guerinot, 2009). The resulting Fe(III)-PS complexes are transported into root cells via transporters of the Yellow stripe like (YSL) family of proteins (Inoue et al., 2009; Lee et al., 2009a). Nicotianamine (NA), which is synthesized by 
nicotianamine synthase (NAS) from S-adenosyl-L-methionine, is a ubiquitous metal chelator in plants and regulates iron translocation within and between cells and transports it to veins, flowers, and seeds (Takahashi et al., 2003). NA also serves as a substrate for nicotianamine aminotransferase (NAAT) to produce a $3^{\prime \prime}$-oxo intermediate and subsequently, DMA is synthesized by deoxymugineic acid synthase (DMAS) (Haydon and Cobbett, 2007; Kim and Guerinot, 2007). Six members of OsNAAT family have been identified in rice plants, however, only OsNAAT1 is regulated by plant iron status (Inoue et al., 2008). OsDMAS1 is also up-regulated in both root and shoots under iron-deficient condition (Bashir et al., 2006; Bashir and Nishizawa, 2006). In addition to the iron uptake using phytosiderophores/DMA, rice also possesses an Fe(II) uptake system. OsIRT1 and OsIRT2, the homologs of iron-regulated transporter IRT1 in Arabidopsis, are specifically up-regulated in roots of iron-deficient rice plants (Ishimaru et al., 2006).

Once iron is loaded into the xylem, the chelators such as citrate, NA, and DMA are required for further transport in the plant (Jeong and Guerinot, 2009). In rice, a ferric reductase defective (FRD) 3-like gene, OsFRDL1, is involved in iron-citrate translocation from rice roots to shoots (Yokosho et al., 2009). Transporters that are encoded by the YSL family of genes, OsYSL2, OsYSL15, OsYSL16, OsYSL18, are also involved in long distance transport of DMA-Fe(III) and/or NA-Fe(II) complexes (Aoyama et al., 2009; Inoue et al., 2009; Ishimaru et al., 2010; Kakei et al., 2012; Zheng et al., 2012). However, much still remains to be unraveled about intracellular metal transport involving vacuoles, chloroplasts, and mitochondria, although some transporters have been identified with specific iron translocation roles for these compartments. An iron deficiency-inducible mitochondrial iron-regulated gene (OsMIR; Ishimaru et al., 2009) and mitochondrial iron transporter $(O s M I T)$, whose expression increases under excessive iron condition, were identified in rice (Bashir et al., 2011). Permease in chloroplasts 1 (OsPIC1) is also associated with chloroplast iron transport, while the vacuolar membrane localized OsVIT1 and OsVIT2 (Vacuolar iron transporter 1 and 2) mediate sequestration of $\mathrm{Fe}(\mathrm{II}), \mathrm{Zn}(\mathrm{II})$, and $\mathrm{Mn}(\mathrm{II})$ into vacuoles, with OsVIT2 being very responsive to Fe treatments (Zhang et al., 2012). Conversely, transporters of the Natural Resistance Associated Macrophage Protein (NRAMP) family appear to have important roles in mobilizing export of vacuolar Fe stores (Lanquar et al., 2005). Despite these advancements, the coordinated function of different transporters that have a role in iron homeostasis is not fully understood.

Strategies to improve iron content in rice grains were mostly targeted at effective iron (Fe) uptake from the soil and translocation in the plant, in addition to directing $\mathrm{Fe}$ into the rice endosperm. Most of the strategies used NAS and ferritin, a protein that stores iron in a bioavailable form (Lonnerdal et al., 2006; Jin et al., 2009). Endosperm-specific expression of ferritin or the constitutive expression of NAS mostly achieved around 2 - to 3-fold increases of iron in the endosperm (Goto et al., 1999; Lucca et al., 2001; Vasconcelos et al., 2003; Qu et al., 2005; Lee et al., 2009b, 2012). A 4.2-fold increase in Fe content was reported in plants over-expressing OsNAS2 under the control of the CaMV35S promoter (Johnson et al., 2011). The possibility of using other transporters for improving endosperm iron content has also been explored recently. Specific expression of OsYSL2 in the vascular tissue and around the endosperm lead to a 4.4fold increase of iron concentration in the polished rice grains (Ishimaru et al., 2010). Over-expression of OsIRT1 under the control of the maize ubiquitin promoter also increased Fe concentration to $113 \%$ compared to wild type grains (Lee and An, 2009). Alternatively, a few studies focused on the endosperm-specific expression of Phytases (Lucca et al., 2001). These enzymes can degrade phytate, a chelating agent that binds iron as well as other metals and store them in a non-bioavailable form for human consumption within the grain (Brinch-Pedersen et al., 2002).

The overexpression of multiple genes through a single construct, i.e., barley NAS expressed under rice actin promoter, soybean ferritin duplicated and expressed under two different endosperm specific promoters as well as rice OsYSL2 duplicated and expressed under endosperm specific and sucrose transporter promoters, resulted in 4.4-fold increase of iron in polished grains of field grown T3 rice plants (Masuda et al., 2012). In another approach, Wirth and collaborators reported a more than 6-fold increase in endosperm of rice plants constitutively expressing A. thaliana NAS (AtNAS), together with endospermspecific expression of Phaseolus vulgaris ferritin and Aspergillus fumigates phytase as a single construct (NFP rice; Wirth et al., 2009). The effect of NAS and ferritin genes was synergistic in these plants, indicating that none of the iron uptake, transport, or storage systems in the engineered rice plants were saturated.

Here, we investigated the molecular impact of the transgenes on the expression of endogenous iron homeostasis-related genes in the engineered NFP rice plants. We performed targeted expression profiling of 28 genes involved in iron homeostasis. Our data suggests that the transgenes did not interfere with endogenous iron homeostasis at large, but modulated the expression of a few genes to facilitate iron uptake, translocation, and storage. The results provide new insights into coordinated role of different genes, particularly those involved in phytosiderophore synthesis and iron translocation, in maintaining iron homeostasis within the NFP plants while transporting more iron to the grains in these plants.

\section{RESULTS}

The relative expression levels of 28 endogenous rice genes related to iron (or metal) homeostasis (Table 1) were analyzed in transgenic NFP plants and non-transgenic control plants (NTS). The genes studied included those involved in NA and DMA synthesis, the YSL transporters, the iron-regulated transporters, genes from zinc-regulated transporter IRT-like proteins (ZIP) family, transcription factors, as well as the inter- and intra-cellular transporters. The plants were subjected to sufficient and deficient iron availability conditions and the expression levels of selected genes were studied in flag leaf, root, and grain samples collected at three grain development stages, i.e., milky, dough, and mature. In order to select for the reference genes which could be used for all the different sample types and growth stages, a preliminary test with at least 13 genes selected from the gene expression database, Genevestigator ${ }^{\text {TM }}$ (Zimmermann et al., 2005) as well as 
Table 1 | List of genes tested for their expression pattern in the NFP plants in comparison to their non-transgenic siblings.

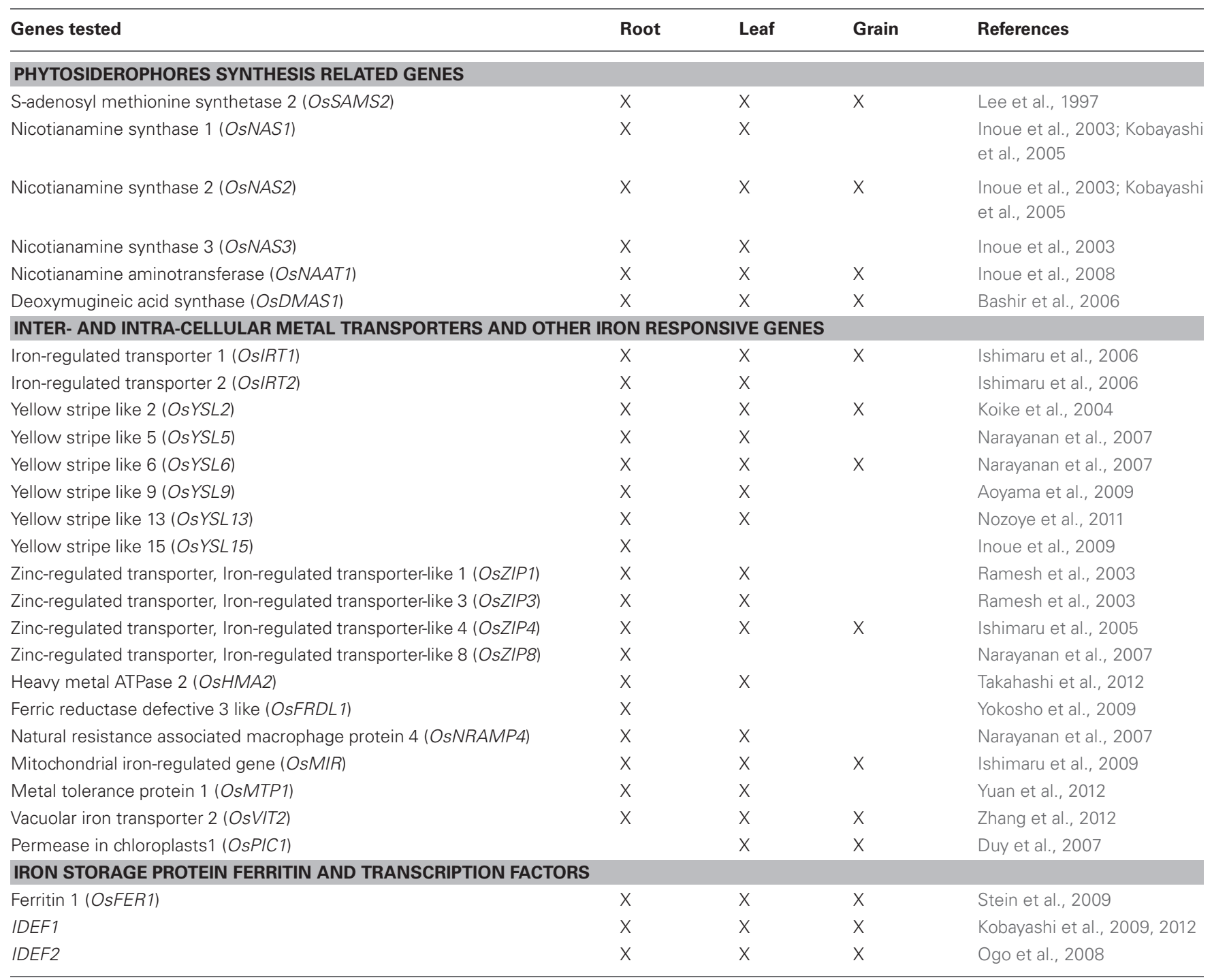

The symbol " $X$ " marks the tissue (root, flag leaf, or grain) in which the corresponding gene was tested.

literature were tested. The genes with medium to high expression in all rice tissues were chosen from Genevestigator ${ }^{\mathrm{TM}}$ for the pilot qRT-PCR test. Among these tested genes, IWS1 C-terminus family protein (LOC_Os01g05420) and ATP binding protein (LOC_Os11g43970.1) ranked among the best five genes identified in our analysis and were therefore used in the experiment. The data from LOC_Os01g05420 expression was used for normalization of real-time quantitative expression of the test genes. The observed changes in the expression patterns of the tested genes in the NFP plants in comparison to their non-transgenic siblings (NTS plants) are summarized below.

\section{EXPRESSION PROFILES OF GENES INVOLVED IN PHYTOSIDEROPHORE SYNTHESIS AND IRON UPTAKE IN ROOTS}

Six rice genes encoding enzymes in phytosiderophore synthesis, and thus involved in iron uptake as well as in iron translocation, were studied. The genes included S-adenosyl-1-methionine synthetase 2 (OsSAMS2), the NAS family members OsNAS1,
OsNAS2 and OsNAS3, OsNAAT1, as well as OsDMAS1. The genes (except OsNAS3) are predominantly expressed in roots and induced by low Fe availability as a part of the Fe deficiency response that serves to increase Fe acquisition (Figures 1-3, A1). As could be expected, over-expression of AtNAS in NFP plants resulted in higher expression of OsSAMS2, OsNAS1, OsNAS3, and OsDMAS1 as compared to the NTS plants, primarily under iron-deficient conditions (Figure A1).

OsSAMS2 was expressed at higher levels during Fe deficiency (mainly in the early development stages) in both NFP and NTS plants, but at the milky stage of grain filling the NFP roots showed further 1.7-fold higher expression of OsSAMS2 (Figure A1, Table 2) than NTS roots. At other stages and in other tissues tested, the NFP and NTS plants did not differ significantly for OsSAMS2 expression. Among the NAS genes, OsNAS3 was overall expressed at low levels compared to OsNAS1 and OsNAS2, but its expression in NFP roots was significantly increased at mature stage under high iron condition and at the milky stage 

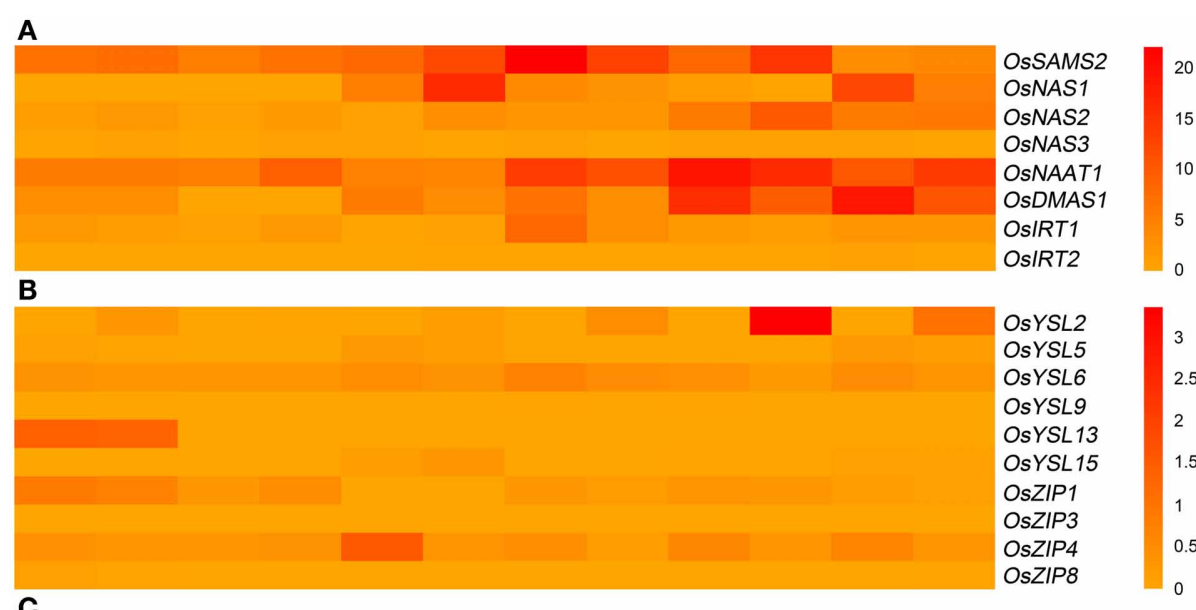

C
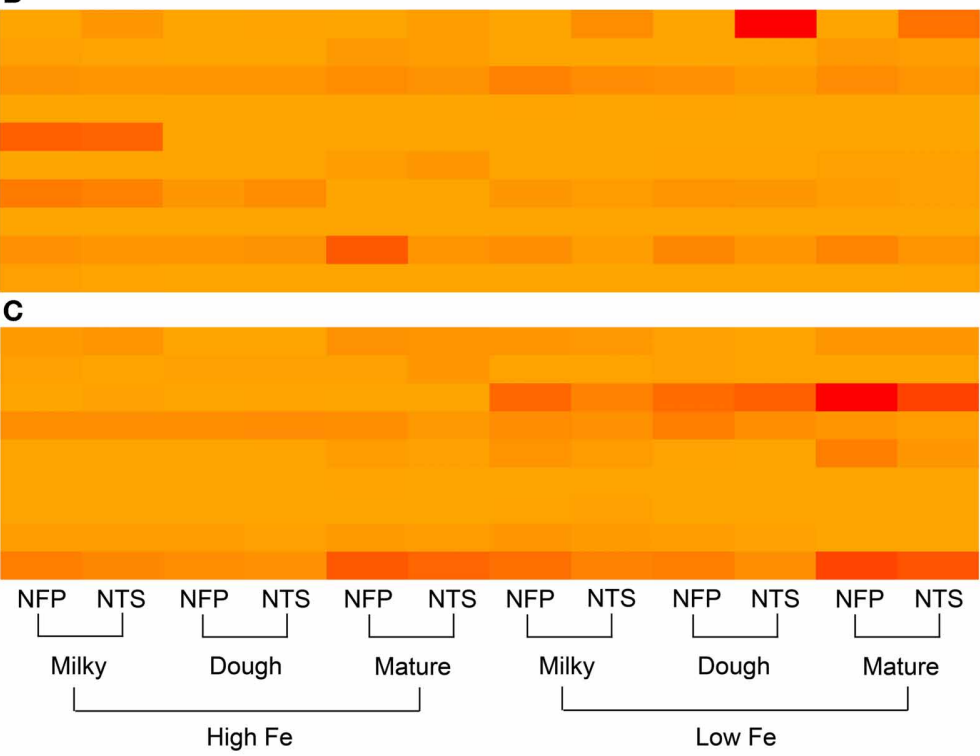

FIGURE 1 | Overall view of genes that are differentially expressed in NFP roots as compared to NTS roots over different plant development stages (corresponding to grain filling stages-milky, dough, and mature) and upon different iron supplies (high and low Fe). (A) Expression levels of genes involved in nicotianamine and deoxymugeneic acid synthesis as well as the iron-regulated transporters. (B) Expression levels of genes belonging to yellow stripe like family of transporters and the transporters belonging to zinc-regulated transporter IRT-like proteins (ZIP) family. (C) Expression levels of transcription factors OsIDEF1 and OsIDEF2 regulating iron homeostasis as well as other inter-and intra-cellular metal transporters. Data represents the mean of three biological replicates. Note the differences in scale between panels (A), (B), and (C) for relative expression levels of tested genes. in the plants grown in iron-deficient conditions (Figure A1). In the mature stage of grain filling, OsNAS1 was up-regulated in NFP roots (2.3-fold) as compared to NTS roots. OsDMAS1 was generally up-regulated in iron-deficient conditions in both the genotypes, but at the milky and mature stages of grain filling, its expression was 2-fold and 1.8-fold higher in the NFP roots than NTS roots, respectively (Figure A1, Table 2). A significant increase of OsDMAS1 expression could also be detected in NFP grains at the milky stage in iron-deficient conditions. These results suggest that the genes involved in NA and DMA synthesis are coordinately regulated in NFP plants, which contributed to increased iron uptake and facilitated translocation within these plants.

\section{OTHER GENES INVOLVED IN IRON UPTAKE AND IRON TRANSLOCATION WITHIN THE PLANT}

The two iron-regulated transporters OsIRT1 and OsIRT2, as well as several members of the YSL and zinc-regulated transporter IRT-like proteins (ZIP) family were studied, including OsYSL2, OsYSL5, OsYSL6, OsYSL9, OsYSL13, OsYSL15 among YSLs and ZIP1, ZIP3, ZIP4, and ZIP8 from the ZIP family (Figures 1-3).
Expression of OsIRT1 and OsIRT2 were mainly induced during iron deficiency and particularly in the roots, with OsIRT1 expressed at higher levels than OsIRT2 as was previously reported (Ishimaru et al., 2006). Further, OsIRT1 was expressed 2.6-fold higher in NFP roots than NTS roots at the milky stage of grain filling (Figure A2, Table 2), while OsIRT2 expression was not significantly different in NFP and NTS plants. OsIRT1 was also significantly up-regulated in the grains (1.5-fold) and leaves (3.5fold) of NFP plants growing under sufficient iron conditions, both at milky and mature stages of grain filling, respectively, but expression levels were generally low in these tissues.

Among the YSL genes, significant transcript level differences were detected for OsYSL2 and OsYSL6, while OsYSL5, OsYSL9, OsYSL13, and OsYSL15 showed no or negligible expression differences between NFP and the NTS plants (Figures 1-3). OsYSL2, a transporter of the NA-Fe(II) complex, is induced during iron deficiency (Ishimaru et al., 2010), which is also the case in NTS plants where OsYSL2 is significantly up-regulated in leaves at the milky stage and in the roots at the dough stage of grain filling (Figure A3, Table 2). However, the OsYSL2 expression remained unchanged in NFP plants grown in low iron conditions. In contrast, a significant overexpression of OsYSL6 was observed in 


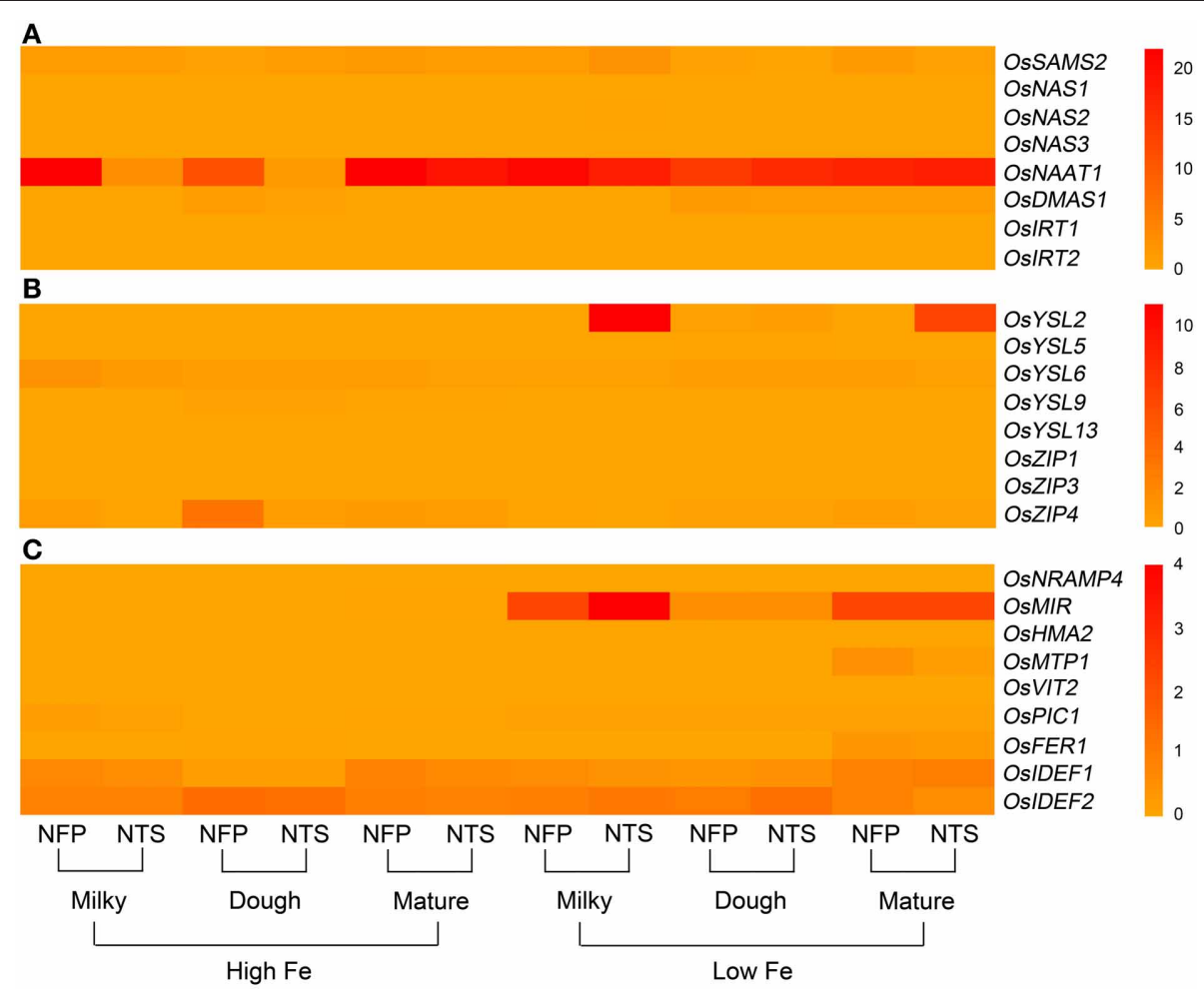

FIGURE 2 | Overall view of genes that are differentially expressed in NFP flag leaves as compared to NTS flag leaves over different plant development stages (corresponding to grain filling stages-milky, dough, and mature) and upon different iron supplies (high and low Fe). (A) Expression levels of genes involved in nicotianamine and deoxymugeneic acid synthesis as well as the iron-regulated transporters. (B) Expression levels of genes belonging to yellow stripe like family of transporters and the transporters belonging to zinc-regulated transporter IRT-like proteins (ZIP) family. (C) Expression levels of transcription factors OsIDEF1 and OsIDEF2 regulating iron homeostasis as well as other inter- and intra-cellular metal transporters. Data represents the mean of three biological replicates. Note the differences in scale between panels (A), (B), and (C) for relative expression levels of tested genes.
NFP grains at maturity and a slightly increased expression in the roots at the dough stage of grain filling in iron-deficient conditions, as compared to NTS plants. Also, OsYSL6 was up-regulated in NFP flag leaves at the milky stage of grain filling as compared to NTS leaves in iron-sufficient conditions. OsYSL6 has been suggested as a Mn-NA transporter and also to play a role in detoxification of high $\mathrm{Mn}$ in roots and shoots (Sasaki et al., 2011). NFP and NTS plants contain similar Mn concentrations, except for some increase in polished and brown grains in irondeficient conditions (Fe $20 \mu \mathrm{M}$; Wirth et al., 2009). It is possible that the induced expression of OsYSL6 in NFP plants contributes to the small increase of Mn in NFP grains when iron availability is low.

OsZIP4 was up-regulated in NFP leaves at dough stage of grain filling (6.3-fold) and in roots at grain maturity (4.2-fold) in ironsufficient conditions. In iron-deficient conditions, NFP grains at maturity and NFP roots at the dough stage of grain filling had 3.3-fold and 2.1-fold higher expression of OsZIP4 as compared to NTS plants, respectively (Figure A3, Table 2). OsZIP1 was also up-regulated in low iron conditions in NFP roots at the dough stage (1.3-fold) and leaves at the milky stage of grain filling (2.5-fold). In addition, an approximate 6-fold higher expression was detected at the milky and mature stages of grain filling in the leaves of NFP plants grown with sufficient iron supply. However, it should be noted that OsZIP1 is expressed only weakly in the leaves. OsZIP3 and OsZIP8 were expressed at even lower levels than OsZIP1, and their expression profiles were not significantly different between the genotypes, except for up-regulated OsZIP3 expression in NFP roots at the dough stage of grain filling and down regulation in leaves at the milky stage of grain filling in low Fe conditions, as compared to NTS plants (Table 2). The upregulation of OsZIP1 and OsZIP4 under high iron conditions suggests that NFP plants signaled zinc deficiency when external iron concentration was high. Nevertheless, NFP plants perform better than the NTS plants in terms of zinc content in leaves and grains (Wirth et al., 2009) at both low and high iron conditions.

\section{TRANSCRIPTION FACTORS AND OTHER INTER- AND INTRA-CELLULAR TRANSPORTERS}

The transcription factors encoded by OsIDEF1 and OsIDEF2 are known to be constitutively expressed and not affected by Fe deficiency (Kobayashi et al., 2007; Ogo et al., 2008). Similar expression patterns were obtained in our experiments, with an exception of OsIDEF2, which responded to iron deficiency in NFP plants. OsIDEF2 expression was increased by 1.5 - and 1.8 -fold 


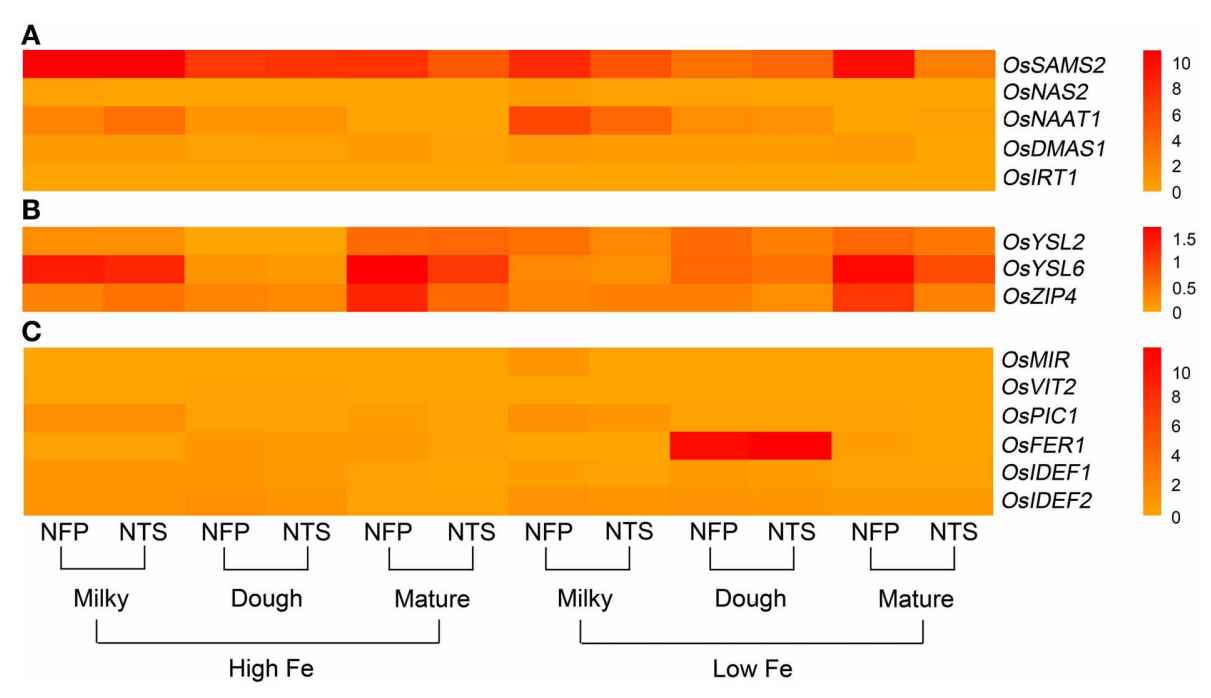

FIGURE 3 | Overall view of genes that are differentially expressed in NFP grains as compared to NTS grains over different plant development stages (corresponding to grain filling stages - milky, dough, and mature) and upon different iron supplies (high and low Fe). (A) Expression levels of genes involved in nicotianamine and deoxymugeneic acid synthesis as well as the iron-regulated transporters. (B) Expression levels of genes belonging to yellow stripe like family of transporters and the transporters belonging to zinc-regulated transporter IRT-like proteins (ZIP) family. (C) Expression levels of transcription factors OsIDEF1 and OsIDEF2 as well as other inter- and intra-cellular metal transporters. Data represents the mean of three biological replicates. Note the differences in scale between panels (A), (B), and (C) for relative expression levels of tested genes. in NFP roots at milky and dough stages of grain filling as compared to NTS roots (Figure A2, Table 2). This up-regulation of OsIDEF2 in NFP roots perhaps reinforced the Fe deficiency signal and thus led to up-regulation of genes involved in Fe translocation. OsIDEF2 is known to be dominantly expressed in vascular bundles in the roots (Kobayashi et al., 2010b).

The mitochondrial iron-regulated (OsMIR) gene was mainly expressed upon iron deficiency. In comparison to the NTS plants, NFP grains had elevated expression of OsMIR at milky and dough stages of grain filling in iron-deficient conditions and at the milky stage of grain filling with sufficient iron availability. However, OsMIR was less induced in NFP leaves than the NTS leaves at the milky stage of grain filling (Figure A2, Table 2), but no significant expression differences were found at other stages. OsHMA2, OsPIC1, OsMTP1, OsNRAMP4, OsFRDL1, OsFER1, and OsVIT2 did not show any significant differences between NFP and NTS plants (Figures 1-3). Only in iron-deficient conditions, NFP mature grains showed a higher expression of OsPIC1 and NFP roots had higher OsHMA2 expression at the dough stage of grain filling when compared to NTS plants (Table 2). OsVIT2 was mostly expressed under sufficient iron conditions, with low expression detected under iron deficiency in roots and leaves. OsVIT1 does not respond to Fe starvation while OsVIT2 was found to be down-regulated in rice roots and shoots (Zhang et al., 2012).

The summary of genes that are differentially regulated in the NFP plants as compared to NTS plants, under Fe-deficient conditions is presented in Figure 4.

\section{DISCUSSION}

The combined overexpression of NAS and endosperm-specific expression of ferritin have a synergistic effect in increasing the iron content in the endosperm of NFP grains (Wirth et al., 2009). In the greenhouse, NFP plants show normal agronomic performance (e.g., plant height, tiller number, grain yield) and perform better under low Fe conditions than NTS plants (Wirth et al., 2009), suggesting that expression of AtNAS1 and ferritin promoted iron increase in the endosperm without interfering with Fe homeostasis in NFP plants.

NA and DMA levels in plants are regulated by NAS and NAAT genes, and both NA and DMA are involved in Fe distribution in plants (Aoyama et al., 2009; Kakei et al., 2009). DMA/phytosiderophore synthesis involves successive reactions that are catalyzed by SAMS, NAS, NAAT, and DMAS (Bashir et al., 2010). OsNAS1, OsNAS2, and OsNAS3 were proposed to perform different physiological functions in response to Fe deficiency (Inoue et al., 2003). Expression of OsNAS1 and OsNAS2, both located on chromosome 3 , is induced in rice roots exposed to Fe deficiency. Thus, a main role of these enzymes in NA synthesis appears to be the increased production of phytosiderophores in iron-deficient roots (Inoue et al., 2003). Expression of OsNAS3, which is located on chromosome 7, was found confined to pericycle cells close to protoxylem and companion cells, and was suggested to play rather limited role in phytosiderophore secretion from roots. Nevertheless, the OsNAS3 protein was shown to catalyze the trimerization of SAM to form NA (Inoue et al., 2003).

During low iron availability, expression of OsSAMS2 and OsNAS3 was significantly up-regulated at the milky stage of grain filling while OsNAS1 had elevated expression levels at maturity in NFP roots when compared to NTS roots. This suggests that under iron deficiency, AtNAS1 overexpression together with the enhanced expression of OsNAS3 and OsNAS1 resulted in increased NA synthesis at milky and mature stages of grain filling. 


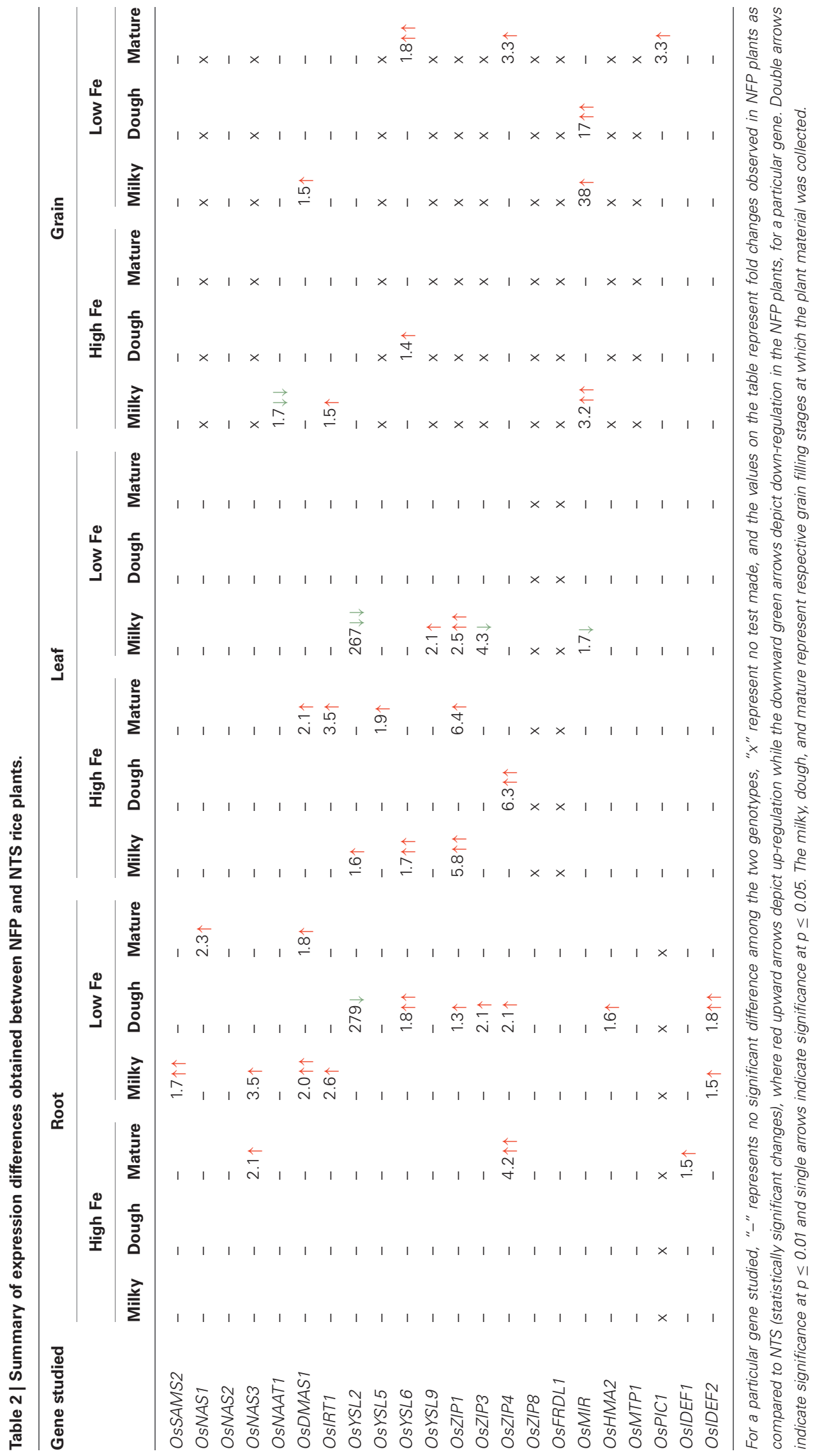




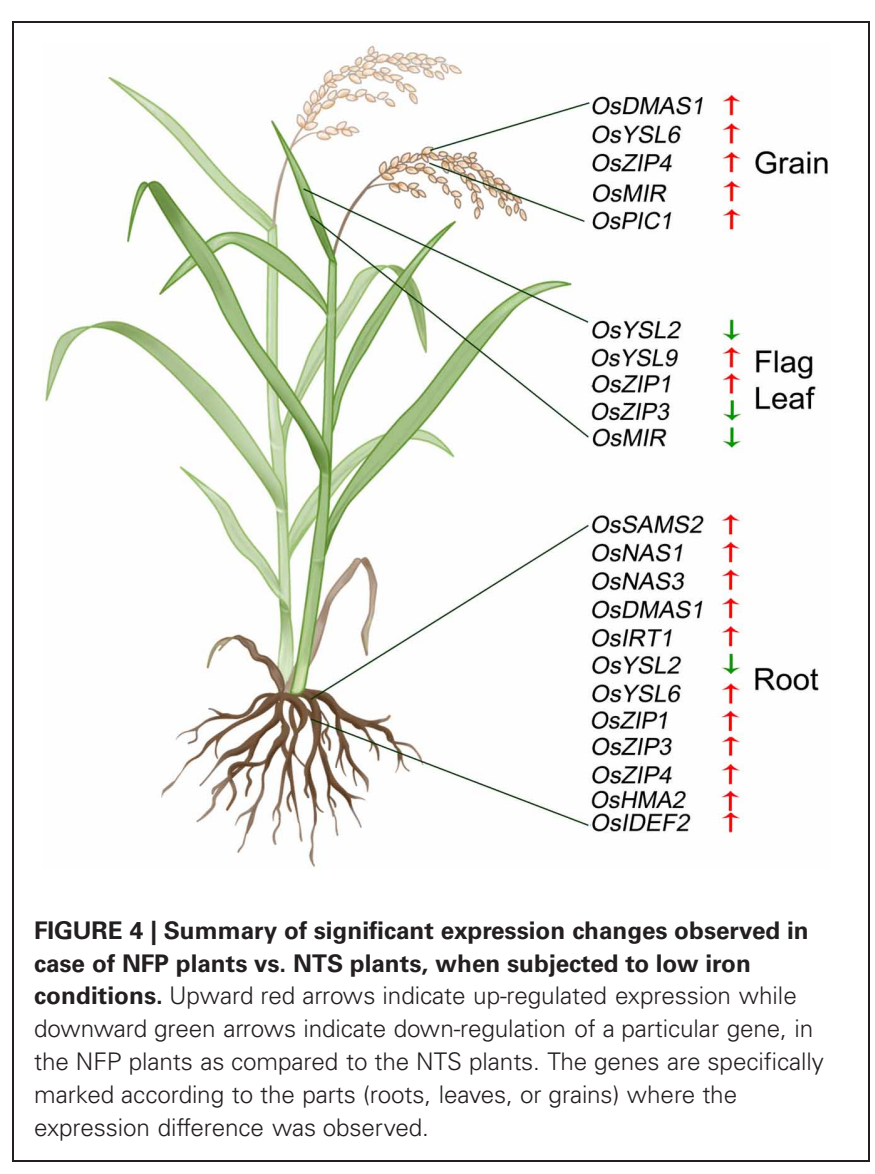

NFP plants produce more NA under iron deficiency (Wirth et al., 2009). Since NA serves as the precursor for DMA, this increase in NA production most likely contributed to increased DMA content in NFP plants. Increased DMA production in NFP plants is further supported by the up-regulation of OsDMAS1 in NFP roots, both at milky and mature stages of grain filling (Figure A1), as well as with increased DMA content in NFP leaves as compared to control plants (unpublished data). OsNAS3 expression is also higher in NFP roots grown at high iron conditions, particularly at maturity. Together, these results suggest that the increased production of NA and DMA in NFP plants facilitated uptake of iron in roots and improved $\mathrm{Fe}$ translocation in the plants. In the roots of iron-deficient plants, OsNAS1-3, OsNAAT1, and OsDMAS1 have similar expression patterns, with strong induction in pericycle cells adjacent to protoxylem (Inoue et al., 2003; Bashir et al., 2006; Inoue et al., 2008). Rice plants have increased DMA concentration in the xylem in iron-deficient conditions (Kakei et al., 2009). The specific contribution of OsNAS3 to iron homeostasis has been previously reported (Lee et al., 2009b), where OsNAS3 activation resulted in increased $\mathrm{Fe}$ and $\mathrm{Zn}$ concentration in the rice grains as well as increased tolerance to heavy metals. This increased Fe concentration is also well-correlated with increased NA and DMA content in plants that have increased OsNAS3 expression (Lee et al., 2009b). Johnson et al. (2011) also showed a positive correlation between increased NA content and increased grain iron content in rice plants overexpressing either of OsNAS1, OsNAS2, and OsNAS3.
Furthermore, the demand for methionine is increased in iron-deficient plants in order to support the increased production of NA and then subsequently DMA. In our experiment, OsSAMS2 was significantly up-regulated in roots of iron-deficient NFP and NTS plants, with a further expression increase in NFP roots at the milky stage of grain filling as compared to NTS plants (Figure A1). This increase of OsSAMS2 expression would be expected to meet the demand for SAM, which is an immediate precursor for NA synthesis. Increased expression of genes participating in the methionine cycle in the roots of iron-deficient wheat, rice, and barley has been reported earlier (Ma et al., 1995; Negishi et al., 2002; Kobayashi et al., 2005).

In iron-deficient conditions, Fe needs to be effectively transported from roots to the shoots via the xylem, and then between cells. Significant advances have been made in identifying transporters involved in iron translocation. However, our understanding of the exact contribution of each one of these transporters in metal flux is rather limited. To date, the principal chelators known to bind iron include citrate (Rellan-Alvarez et al., 2010), NA and DMA (Takahashi et al., 2003; Aoyama et al., 2009; Kakei et al., 2009). The major role of DMA was initially considered to be in iron uptake from the rhizosphere, but several lines of evidence support a chelating role of DMA in both xylem and phloem (Aoyama et al., 2009; Kakei et al., 2009). Based on the expression of OsDMAS1 it has been proposed that DMA is synthesized in the phloem companion cells (Inoue et al., 2008). High concentrations of DMA have been detected in phloem sap in independent studies advocating the involvement of DMA in long distance iron transport (Nishiyama et al., 2012). Several transporters belonging to YSL family could be transporting these chelatorbound iron complexes to other parts of the plant. OsYSL15 and OsYSL18 transport Fe(III)-DMA complexes and are involved in internal translocation of iron (Aoyama et al., 2009; Inoue et al., 2009), while OsYSL2 transports Fe(II)-NA and Mn(II)-NA complexes, but not Fe(III)-DMA (Koike et al., 2004; Ishimaru et al., 2010).

OsYSL2 is induced by iron-deficiency and may be actively involved in long distance phloem transport of Fe(II)-NA complexes in the plant and into the grains (Koike et al., 2004; Ishimaru et al., 2010). Rice plants with reduced OsYSL2 function (RNAi-OsYSL2) have reduced Fe and Mn concentrations in the grains (Ishimaru et al., 2010). Consistent with previous reports (Ishimaru et al., 2010), NTS plants showed induced expression of OsYSL2 upon iron deficiency, but the gene was not up-regulated in NFP roots and leaves under the same condition (Figure A3). In the grains, however, the expression of OsYSL2 increased in NFP plants as well but was not significantly different than in NTS grains. A plausible explanation for this result could be that expression of OsYSL2 is regulated by the endogenous iron status of the plants. It has also been suggested that OsIDEF2 directly regulates expression of OsYSL2 (Ogo et al., 2008). However, the up-regulation of OsIDEF2 in iron-deficient NFP roots (Figure A2) did not lead to increased OsYSL2 expression. It is possible that OsIDEF2 also senses cellular iron status in order to induce the iron-deficiency responsive genes, as was suggested for OsIDEF1 which binds directly to 
divalent metals for sensing cellular metal ion balance (Kobayashi et al., 2012). Importantly, the NFP plants had higher iron content in the grains as compared to NTS plants. It is also possible that NFP plants deployed alternate modes of iron transport to grains than the transfer by OsYSL2 and that the function of OsYSL2 is complemented by another transporter. Our results also reflect effective crosstalk between molecular components involved in Fe homeostasis in different growth conditions and during development to meet the needs for Fe in the plant.

OsMIR, a recently evolved rice-specific mitochondrial gene, is strongly induced under iron deficiency (Ishimaru et al., 2009). NFP leaves had lower expression levels of OsMIR as compared to NTS leaves under low iron conditions, but the gene was upregulated in NFP grains (Figure A2). Since mitochondrial Fe regulation is poorly understood, it is difficult to predict how and to what extent these expression differences contributed to higher grain iron content in the NFP plants. In addition, NFP leaves also had increased expression of OsZIP1 and OsZIP4 under high iron conditions, indicating that NFP plants might signal zinc deficiency when external iron concentration is high. Although OsZIP4 is regulated by zinc (Ishimaru et al., 2005), there exists a strong crosstalk between $\mathrm{Zn}$ and Fe homeostasis in plants. Fe concentrations doubled in Zn-deficient roots (Ishimaru et al., 2005) and plants overexpressing OsZIP4 had significantly increased $\mathrm{Fe}$ in the shoots and roots, in addition to the $\mathrm{Zn}$ increases (Ishimaru et al., 2007). This demonstrates the coordination of $\mathrm{Fe}$ and $\mathrm{Zn}$ homeostasis in plants and also that the expression of OsZIP1 and OsZIP4 are affected by the external supply of iron to the plants (as in the case of both NFP and NTS plants) as well as by endogenous $\mathrm{Fe}$ nutritional status of plants (further increases in NFP plants). Nevertheless, NFP plants had a similar zinc content in the leaves as compared to the controls over a range of tested external iron concentrations and outperformed NTS plants in terms of zinc content in the grains (Wirth et al., 2009). Therefore, $\mathrm{Zn}$ homeostasis is also unaffected in NFP plants.

Together, the increased production of NA and DMA in NFP plants facilitated iron uptake from the rhizosphere as well as effective internal translocation. Expression of several transporter genes appear to be adjusted in the NFP plants in order to utilize overproduced NA and DMA, and the expanded sink for iron storage in the grains via ferritin. However, these adjustments did not interfere with Fe homeostasis in the NFP plants. Further investigations focused on iron speciation in the grain, i.e., $\mathrm{Fe}(\mathrm{II})$ or $\mathrm{Fe}(\mathrm{III})$, and the relative abundance of two forms together with the information on molecules chelated to these forms, will be required for elucidating the exact mechanisms of iron translocation to the grains in NFP plants.

\section{MATERIALS AND METHODS PLANT MATERIAL}

The NFP plants and their non-transgenic siblings (NTS) were grown under greenhouse conditions, in the hydroponics system. The NFP plants are the Oryza sativa ssp. japonica cv. Taipei 309 transformed with Arabidopsis Nicotianamine Synthase gene, Phaseolus vulgaris Ferritin, and A. fumigatus Phytase gene (NFP plants; Wirth et al., 2009). Solutions for the hydroponic system were prepared according to the protocol modified from Kobayashi et al. (2005), using $0.70 \mathrm{mM} \mathrm{K}_{2} \mathrm{SO}_{4}, 0.10 \mathrm{mM}$ $\mathrm{KCl}, 0.10 \mathrm{mM} \mathrm{KH} \mathrm{PO}_{4}, 2.0 \mathrm{mM} \mathrm{Ca}\left(\mathrm{NO}_{3}\right)_{2}, 0.50 \mathrm{mM} \mathrm{MgSO}_{4}$, $10 \mu \mathrm{M} \mathrm{H} \mathrm{H}_{3} \mathrm{BO}_{3}, 0.50 \mu \mathrm{M} \mathrm{MnSO}_{4}, 0.20 \mu \mathrm{M} \mathrm{CuSO}_{4}, 0.01 \mu \mathrm{M}$ $(\mathrm{NH} 4)_{6} \mathrm{Mo}_{7} \mathrm{O}_{24}$, and $0.5 \mu \mathrm{M} \mathrm{ZnSO}$, with different iron concentrations added as $\mathrm{Fe}$ (III)-EDTA according to the treatment (high iron condition: $200 \mu \mathrm{M}$ iron; iron-deficient condition: $10 \mu \mathrm{M}$ iron). Samples were collected at three different grain filling stages: milky stage, dough stage, and mature stage. At the milky stage grains are starting to fill with a white, milky liquid that can be squeezed by pressing the grain between fingers, while in dough stage the milky portion of grain turns into a soft dough and at maturity, the grain is fully developed and hard (parameters as defined in the Rice Knowledge Bank, IRRI, Philippines). At each developmental stage, roots, flag leaves and grains were collected, with at least three biological replicates.

\section{TOTAL RNA EXTRACTION AND cDNA SYNTHESIS}

Total RNA was extracted from the root, flag leaf, and grain samples using Trizol ${ }^{\circledR}$ reagent (Invitrogen, USA) and was treated with DNase I (Thermo Fisher Scientific Inc., USA). RevertAid ${ }^{\mathrm{TM}}$ first strand cDNA synthesis kit (Thermo Fisher Scientific Inc., USA) was used for cDNA synthesis. All steps were carried out following the manufacturers' instructions.

\section{REAL-TIME QUANTITATIVE PCR}

Real-time quantitative PCRs (qRT-PCR) were carried out using Taqman hydrolysis probes (Roche, Switzerland) on 7500 FAST Real Time PCR system (Applied Biosystems, Inc., USA). Total reaction volume of $25 \mu \mathrm{l}$ was used, comprising of $12.5 \mu \mathrm{l}$ mastermix (Applied Biosystems Inc., USA), $1 \mu \mathrm{l}$ cDNA, $2.25 \mu \mathrm{l}$ forward primer and $2.25 \mu \mathrm{l}$ reverse primer, $0.25 \mu \mathrm{l}$ probe (Roche Ltd., Switzerland) and $6.75 \mu \mathrm{l} \mathrm{H}_{2} \mathrm{O}$.

Primers were designed using Roche primer design website (https://www.roche-applied-science.com/sis/rtpcr/upl/index.jsp? $\mathrm{id}=\mathrm{UP} 030000)$. Probe number and primer sequences are presented in the Appendix (Table A1). The Ct value was obtained from 7500 Fast System Software (Applied Biosystems, Inc., USA). The primer efficiency was calculated using LinReg PCR (Tuomi et al., 2010). qRT-PCR data normalization was done as described by Schefe et al. (2006). The obtained data were further analyzed by ANOVA and significant differences between the tested plant materials are presented.

\section{ACKNOWLEDGMENTS}

We thank ETH Zurich (ETH-06 10-3), the Swiss State Secretariat for Education and Research (through COST FA0905), and Ms. Jacqueline Imhof for support of our biofortification work. We also acknowledge the help of our gardener Irene Zurkirchen and of Renato Guidon, Kumar Vasudevan, and Jonghwa Park for help with sample preparations. We acknowledge the contribution of Bernadette Rawyler for the rice plant drawing used in Figure 4 of the article. 


\section{REFERENCES}

Aoyama, T., Kobayashi, T., Takahashi, M., Nagasaka, S., Usuda, K., Kakei, Y., et al. (2009). OsYSL18 is a rice iron(III)-deoxymugineic acid transporter specifically expressed in reproductive organs and phloem of lamina joints. Plant Mol. Biol. 70, 681-692.

Bashir, K., Inoue, H., Nagasaka, S., Takahashi, M., Nakanishi, H., Mori, S., et al. (2006). Cloning and characterization of deoxymugineic acid synthase genes from graminaceous plants. J. Biol. Chem. 281, 32395-32402.

Bashir, K., Ishimaru, Y., and Nishizawa, N. (2010). Iron uptake and loading into rice grains. Rice 3, 122-130.

Bashir, K., Ishimaru, Y., Shimo, H., Nagasaka, S., Fujimoto, M., Takanashi, H., et al. (2011). The rice mitochondrial iron transporter is essential for plant growth. Nat. Commun. 2:322. doi: 10.1038/ncomms 1326

Bashir, K., and Nishizawa, N. K. (2006). Deoxymugineic Acid synthase: a gene important for $\mathrm{Fe}$ acquisition and homeostasis. Plant Signal Behav. 1, 290-292.

Brinch-Pedersen, H., Sorensen, L. D., and Holm, P. B. (2002). Engineering crop plants: getting a handle on phosphate. Trends Plant Sci. 7, 118-125.

Duy, D., Wanner, G., Meda, A. R., Von Wiren, N., Soll, J., and Philippar, K. (2007). PIC1, an ancient permease in Arabidopsis chloroplasts, mediates iron transport. Plant Cell 19, 986-1006.

Goto, F., Yoshihara, T., Shigemoto, N., Toki, S., and Takaiwa, F. (1999). Iron fortification of rice seed by the soybean ferritin gene. Nat Biotechnol. $17,282-286$

Haydon, M. J., and Cobbett, C. S. (2007). Transporters of ligands for essential metal ions in plants. New Phytol. 174, 499-506.

Hurrell, R., and Egli, I. (2010). Iron bioavailability and dietary reference values. Am. J. Clin. Nutr. 91, 1461S-1467S.

Inoue, H., Higuchi, K., Takahashi, M., Nakanishi, H., Mori, S., and Nishizawa, N. K. (2003). Three rice nicotianamine synthase genes, OsNAS1, OsNAS2, and OsNAS3 are expressed in cells involved in longdistance transport of iron and differentially regulated by iron. Plant $\mathrm{J}$. $36,366-381$

Inoue, H., Kobayashi, T., Nozoye, T., Takahashi, M., Kakei, Y., Suzuki, K., et al. (2009). Rice OsYSL15 is an iron-regulated iron(III)deoxymugineic acid transporter expressed in the roots and is essential for iron uptake in early growth of the seedlings. J. Biol. Chem. 284, 3470-3479.

Inoue, H., Takahashi, M., Kobayashi, T., Suzuki, M., Nakanishi, H., Mori, S., et al. (2008). Identification and localisation of the rice nicotianamine aminotransferase gene OsNAAT1 expression suggests the site of phytosiderophore synthesis in rice. Plant Mol. Biol. 66, 193-203.

Institute of Medicine (I. O. M.). (2013). Food and Nutrition Board, Dietary Reference Intakes (DRIs): Estimated Average Requirements. Available online at: http://www.iom.edu/ Activities/Nutrition/SummaryDRIs/ $\sim /$ media/Files/Activity\%20Files/Nu trition/DRIs/New\%20Material/5D RI\%20Values\%20SummaryTables\% 2014.pdf (Accessed February 20, 2013).

Ishimaru, Y., Bashir, K., Fujimoto, M., An, G., Itai, R. N., Tsutsumi, N., et al. (2009). Rice-specific mitochondrial iron-regulated gene (MIR) plays an important role in iron homeostasis. Mol. Plant 2, 1059-1066.

Ishimaru, Y., Masuda, H., Bashir, K., Inoue, H., Tsukamoto, $\mathrm{T}$., Takahashi, M., et al. (2010). Rice metal-nicotianamine transporter, OsYSL2, is required for the longdistance transport of iron and manganese. Plant J. 62, 379-390.

Ishimaru, Y., Masuda, H., Suzuki, M., Bashir, K., Takahashi, M., Nakanishi, H., et al. (2007). Overexpression of the OsZIP4 zinc transporter confers disarrangement of zinc distribution in rice plants. J. Exp. Bot. 58, 2909-2915.

Ishimaru, Y., Suzuki, M., Kobayashi, T., Takahashi, M., Nakanishi, H., Mori, S., et al. (2005). OsZIP4, a novel zinc-regulated zinc transporter in rice. J. Exp. Bot. 56, 3207-3214.

Ishimaru, Y., Suzuki, M., Tsukamoto, T., Suzuki, K., Nakazono, M., Kobayashi, T., et al. (2006). Rice plants take up iron as an $\mathrm{Fe}^{3+}$. phytosiderophore and as $\mathrm{Fe}^{2+}$. Plant J. 45, 335-346.

Jeong, J., and Guerinot, M. L. (2009). Homing in on iron homeostasis in plants. Trends Plant Sci. 14, 280-285.

Jin, F., Frohman, C., Thannhauser, T. W., Welch, R. M., and Glahn, R. P. (2009). Effects of ascorbic acid, phytic acid and tannic acid on iron bioavailability from reconstituted ferritin measured by an in vitro digestion-Caco-2 cell model. $\mathrm{Br}$. J. Nutr. 101, 972-981.

Johnson, A. A., Kyriacou, B., Callahan, D. L., Carruthers, L., Stangoulis, J.,
Lombi, E., et al. (2011). Constitutive overexpression of the OsNAS gene family reveals single-gene strategies for effective ironand zinc-biofortification of rice endosperm. PLOS ONE 6:e24476. doi: 10.1371/journal.pone.0024476

Kakei, Y., Ishimaru, Y., Kobayashi, T. Yamakawa, T., Nakanishi, H., and Nishizawa, N. K. (2012). OsYSL16 plays a role in the allocation of iron. Plant Mol. Biol. 79, 583-594.

Kakei, Y., Yamaguchi, I., Kobayashi, T., Takahashi, M., Nakanishi, H. Yamakawa, T., et al. (2009). A highly sensitive, quick and simple quantification method for nicotianamine and 2'-deoxymugineic acid from minimum samples using LC/ESITOF-MS achieves functional analysis of these components in plants. Plant Cell Physiol. 50, 1988-1993.

Kim, S. A., and Guerinot, M. L. (2007) Mining iron: iron uptake and transport in plants. FEBS Lett. 581, 2273-2280.

Kobayashi, T., Itai, R. N., Aung, M. S., Senoura, T., Nakanishi, H., and Nishizawa, N. K. (2012). The rice transcription factor IDEF1 directly binds to iron and other divalent metals for sensing cellular iron status. Plant J. 69, 81-91.

Kobayashi, T., Itai, R. N., Ogo, Y., Kakei, Y., Nakanishi, H., Takahashi, M. et al. (2009). The rice transcription factor IDEF1 is essential for the early response to iron deficiency, and induces vegetative expression of late embryogenesis abundant genes. Plant, J. 60, 948-961.

Kobayashi, T., Nakanishi, H., and Nishizawa, N. K. (2010a). Dual regulation of iron deficiency response mediated by the transcription factor IDEF1. Plant Signal Behav. 5 , 157-159.

Kobayashi, T., Ogo, Y., Aung, M. S. Nozoye, T., Itai, R. N., Nakanishi, H., et al. (2010b). The spatial expression and regulation of transcription factors IDEF1 and IDEF2. Ann. Bot. 105, 1109-1117.

Kobayashi, T., and Nishizawa, N. K. (2012). Iron uptake, translocation, and regulation in higher plants. Annu. Rev. Plant Biol. 63, 131-152.

Kobayashi, T., Ogo, Y., Itai, R. N., Nakanishi, H., Takahashi, M., Mori, S., et al. (2007). The transcription factor IDEF1 regulates the response to and tolerance of iron deficiency in plants. Proc. Natl. Acad. Sci. U.S.A. 104, 19150-19155.

Kobayashi, T., Suzuki, M., Inoue, H., Itai, R. N., Takahashi, M. Nakanishi, H., et al. (2005). Expression of iron-acquisitionrelated genes in iron-deficient rice is co-ordinately induced by partially conserved iron-deficiencyresponsive elements. J. Exp. Bot. 56, 1305-1316.

Koike, S., Inoue, H., Mizuno, D., Takahashi, M., Nakanishi, H., Mori, S., et al. (2004). OsYSL2 is a rice metal-nicotianamine transporter that is regulated by iron and expressed in the phloem. Plant $J$. 39, 415-424.

Lanquar, V., Lelievre, F., Bolte, S., Hames, C., Alcon, C., Neumann, D., et al. (2005). Mobilization of vacuolar iron by AtNRAMP3 and AtNRAMP4 is essential for seed germination on low iron. EMBO J. 24, 4041-4051.

Lee, J. H., Chae, H. S., Lee, J. H., Hwang, B., Hahn, K. W., Kang, B. G., et al. (1997). Structure and expression of two cDNAs encoding S-adenosyl-L-methionine synthetase of rice (Oryza sativa L.). Biochim. Biophys. Acta 1354, 13-18.

Lee, S., and An, G. (2009). Overexpression of OsIRT1 leads to increased iron and zinc accumulations in rice. Plant Cell Environ. 32, 408-416.

Lee, S., Chiecko, J. C., Kim, S. A., Walker, E. L., Lee, Y., Guerinot, M. L., et al. (2009a). Disruption of OsYSL15 leads to iron inefficiency in rice plants. Plant Physiol. 150, 786-800.

Lee, S., Jeon, U. S., Lee, S. J., Kim, Y.K., Persson, D. P., Husted, S., et al. (2009b). Iron fortification of rice seeds through activation of the nicotianamine synthase gene. Proc. Natl. Acad. Sci. U.S.A. 106, 22014-22019.

Lee, S., Kim, Y. S., Jeon, U. S., Kim, Y. K., Schjoerring, J. K., and An, G. (2012). Activation of Rice nicotianamine synthase 2 (OsNAS2) enhances iron availability for biofortification. Mol. Cells 33, 269-275.

Lonnerdal, B., Bryant, A., Liu, X., and Theil, E. C. (2006). Iron absorption from soybean ferritin in nonanemic women. Am. J. Clin. Nutr. 83, 103-107.

Lucca, P., Hurrell, R., and Potrykus, I. (2001). Genetic engineering approaches to improve the bioavailability and the level of iron in rice grains. Theor. Appl. Genet. 102, 392-397.

Ma, J. F., Shinada, T., Matsuda, C., and Nomoto, K. (1995). Biosynthesis of phytosiderophores, mugineic acids, associated with methionine cycling. J. Biol. Chem. 270, 16549-16554.

Masuda, H., Ishimaru, Y., Aung, M. S., Kobayashi, T., Kakei, Y., Takahashi, M., et al. (2012). Iron biofortification in rice by the introduction of multiple genes involved in 
iron nutrition. Sci. Rep. 2:543. doi: 10.1038/srep00543

Narayanan, N. N., Vasconcelos, M. W., and Grusak, M. A. (2007). Expression profiling of Oryza sativa metal homeostasis genes in different rice cultivars using a cDNA macroarray. Plant Physiol. Biochem. 45, 277-286.

Negishi, T., Nakanishi, H., Yazaki, J., Kishimoto, N., Fujii, F., Shimbo, K., et al. (2002). cDNA microarray analysis of gene expression during $\mathrm{Fe}$-deficiency stress in barley suggests that polar transport of vesicles is implicated in phytosiderophore secretion in Fe-deficient barley roots. Plant J. 30, 83-94.

Nishiyama, R., Kato, M., Nagata, S., Yanagisawa, S., and Yoneyama, T. (2012). Identification of Zn-nicotianamine and $\mathrm{Fe}-2^{\prime}$ Deoxymugineic acid in the phloem sap from rice plants (Oryza sativa L.). Plant Cell Physiol. 53, 381-390.

Nozoye, T., Nagasaka, S., Kobayashi, T., Takahashi, M., Sato, Y., Sato, Y., et al. (2011). Phytosiderophore efflux transporters are crucial for iron acquisition in graminaceous plants. J. Biol. Chem. 286, 5446-5454.

Ogo, Y., Kobayashi, T., Nakanishi Itai, R., Nakanishi, H., Kakei, Y., Takahashi, M., et al. (2008). A novel NAC transcription factor, IDEF2, that recognizes the iron deficiency-responsive element 2 regulates the genes involved in iron homeostasis in plants. J. Biol. Chem. 283, 13407-13417.

Palmer, C. M., and Guerinot, M. L. (2009). Facing the challenges of $\mathrm{Cu}$, $\mathrm{Fe}$ and $\mathrm{Zn}$ homeostasis in plants. Nat. Chem. Biol. 5, 333-340.

Qu, L. Q., Yoshihara, T., Ooyama, A., Goto, F., and Takaiwa, F. (2005).
Iron accumulation does not parallel the high expression level of ferritin in transgenic rice seeds. Planta 222, 225-233.

Ramesh, S. A., Shin, R., Eide, D. J., and Schachtman, D. P. (2003). Differential metal selectivity and gene expression of two zinc transporters from rice. Plant Physiol. 133, 126-134.

Rellan-Alvarez, R., Giner-MartinezSierra, J., Orduna, J., Orera, I., Rodriguez-Castrillon, J. A., Garcia-Alonso, J. I., et al. (2010). Identification of a tri-iron(III), tri-citrate complex in the xylem sap of iron-deficient tomato resupplied with iron: new insights into plant iron long-distance transport. Plant Cell Physiol. 51, 91-102.

Sasaki, A., Yamaji, N., Xia, J., and Ma, J. F. (2011). OsYSL6 is involved in the detoxification of excess manganese in rice. Plant Physiol. 157, 1832-1840.

Schefe, J. H., Lehmann, K. E., Buschmann, I. R., Unger, T., and Funke-Kaiser, H. (2006). Quantitative real-time RT-PCR data analysis: current concepts and the novel "gene expression's CT difference" formula. J. Mol. Med. (Berl.) 84, 901-910.

Stein, R. J., Ricachenevsky, F. K., and Fett, J. P. (2009). Differential regulation of the two rice ferritin genes (OsFER1 and OsFER2). Plant Sci. $177,563-569$.

Takahashi, M., Terada, Y., Nakai, I., Nakanishi, H., Yoshimura, E., Mori, S., et al. (2003). Role of nicotianamine in the intracellular delivery of metals and plant reproductive development. Plant Cell 15, 1263-1280.

Takahashi, R., Ishimaru, Y., Shimo, H., Ogo, Y., Senoura, T., Nishizawa, N.
K., et al. (2012). The OsHMA2 transporter is involved in rootto-shoot translocation of $\mathrm{Zn}$ and $\mathrm{Cd}$ in rice. Plant Cell Environ. 35, 1948-1957.

Tuomi, J. M., Voorbraak, F., Jones, D. L., and Ruijter, J. M. (2010). Bias in the $\mathrm{Cq}$ value observed with hydrolysis probe based quantitative PCR can be corrected with the estimated PCR efficiency value. Methods 50, 313-322.

Vasconcelos, M., Datta, K., Oliva, N. Khalekuzzaman, M., Torrizo, L., Krishnan, S., et al. (2003). Enhanced iron and zinc accumulation in transgenic rice with the ferritin gene. Plant Sci. 164, 371-378.

Walker, E. L., and Connolly, E. L. (2008). Time to pump iron: irondeficiency-signaling mechanisms of higher plants. Curr. Opin. Plant. Biol. 11, 530-535.

World Health Organization (WHO). (2013). Micronutrient Deficiencies. Available online at: http://www. who.int/nutrition/topics/ida/en/ (Accessed February 20, 2013).

Wirth, J., Poletti, S., Aeschlimann, B., Yakandawala, N., Drosse, B., Osorio, S., et al. (2009). Rice endosperm iron biofortification by targeted and synergistic action of nicotianamine synthase and ferritin. Plant Biotechnol. J. 7, 631-644.

Yokosho, K., Yamaji, N., Ueno, D., Mitani, N., and Ma, J. F. (2009). OsFRDL1 is a citrate transporter required for efficient translocation of iron in rice. Plant Physiol. 149, 297-305.

Yuan, L., Yang, S., Liu, B., Zhang, M., and $\mathrm{Wu}, \mathrm{K}$. (2012). Molecular characterization of a rice metal tolerance protein, OsMTP1. Plant Cell. Rep. 31, 67-79.
Zhang, Y., Xu, Y. H., Yi, H. Y., and Gong, J. M. (2012). Vacuolar membrane transporters OsVIT1 and OsVIT2 modulate iron translocation between flag leaves and seeds in rice. Plant J. 72, 400-410.

Zheng, L., Yamaji, N., Yokosho, K., and Ma, J. F. (2012). YSL16 is a phloem-localized transporter of the copper-nicotianamine complex that is responsible for copper distribution in rice. Plant Cell 24, 3767-3782.

Zimmermann, P., Hennig, L., and Gruissem, W. (2005). Geneexpression analysis and network discovery using Genevestigator. Trends Plant Sci. 10, 407-409.

Conflict of Interest Statement: The authors declare that the research was conducted in the absence of any commercial or financial relationships that could be construed as a potential conflict of interest.

Received: 12 March 2013; accepted: 07 May 2013; published online: 29 May 2013.

Citation: Wang $M$, Gruissem $W$ and Bhullar NK (2013) Nicotianamine synthase overexpression positively modulates iron homeostasis-related genes in high iron rice. Front. Plant Sci. 4:156. doi: 10.3389/fpls.2013.00156

This article was submitted to Frontiers in Plant Physiology, a specialty of Frontiers in Plant Science.

Copyright (๑) 2013 Wang, Gruissem and Bhullar. This is an open-access article distributed under the terms of the Creative Commons Attribution License, which permits use, distribution and reproduction in other forums, provided the original authors and source are credited and subject to any copyright notices concerning any third-party graphics etc. 


\section{APPENDIX}
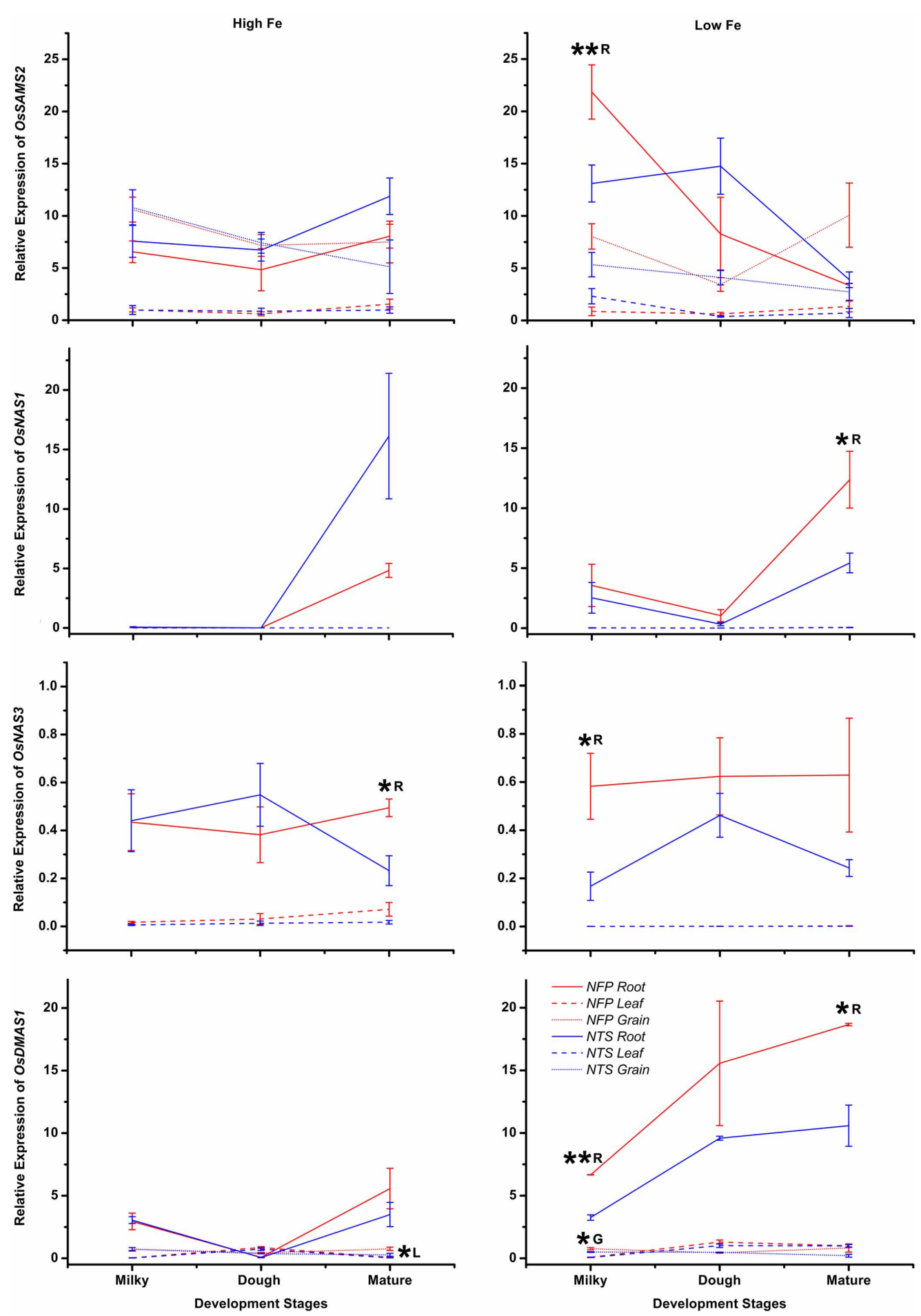

FIGURE A1 | Differences in expression levels for genes involved in nicotianamine and deoxymugeneic acid synthesis (OsSAMS2, OsNAS1, OsNAS3, and OsDMAS1), in the roots, flag leaves, and grains of NFP vs. NTS plants are presented. Values are mean \pm standard error of mean, of the three biological replicates. Significant differences between NFP and NTS tissues (roots, flag

leaves, grains) under a particular treatment (high $\mathrm{Fe}$ or low $\mathrm{Fe}$ ) or at a particular development stage (milky, dough, or mature) are marked with single $\left({ }^{*} p \leq 0.05\right)$ or double asterisks $\left({ }^{* *} p \leq 0.01\right)$, respectively. The letters $R, L$, and $G$ represent root, leaf, and grain, respectively. Note the differences in scale at Y-axis for relative expression levels of tested genes. 

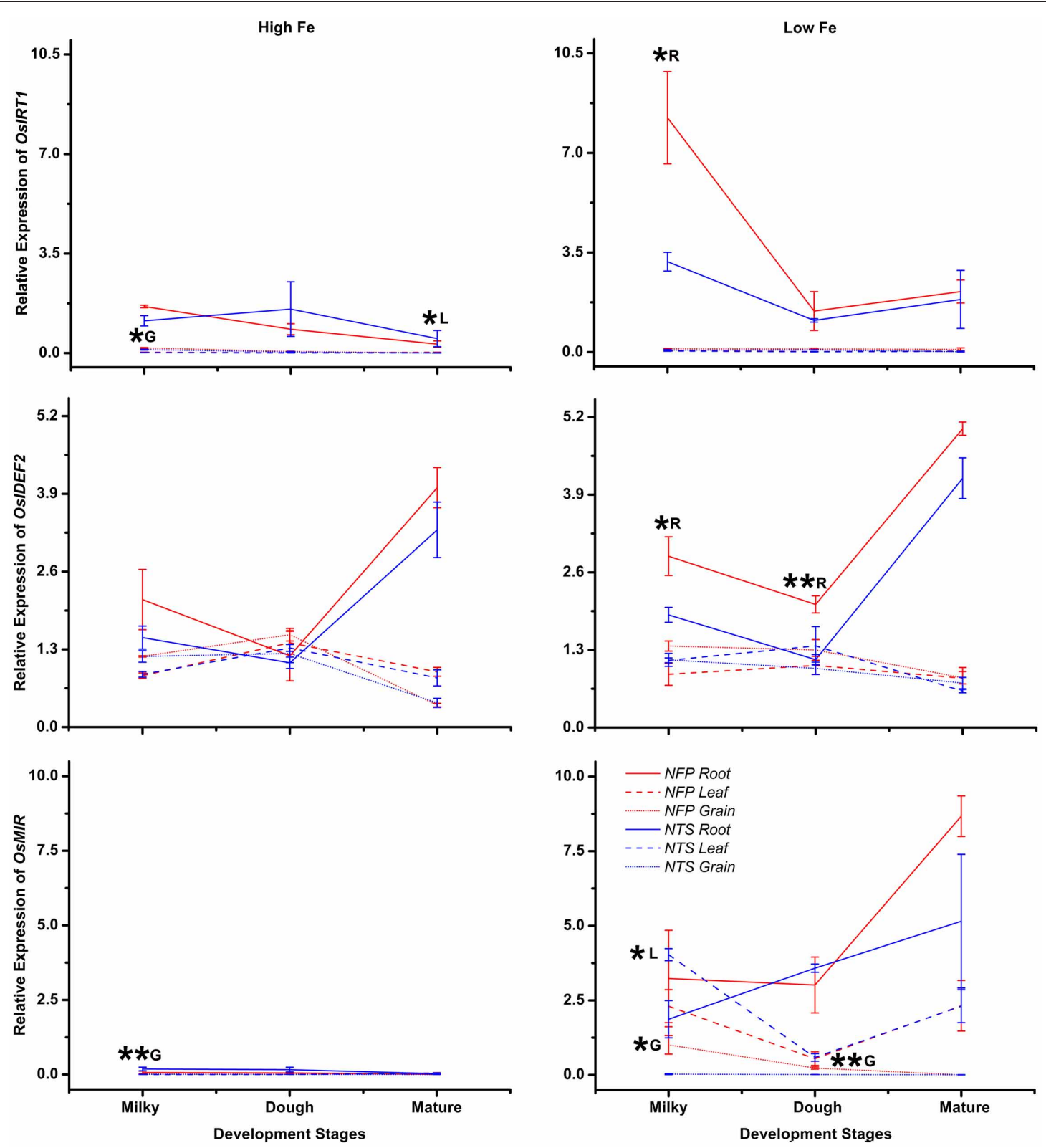

FIGURE A2 | Differences in expression levels of iron-regulated transporter OsIRT1, the transcription factor OsIDEF2, and the mitochondrial iron regulated gene OsMIR, in the roots, flag leaves, and grains of NFP vs. NTS plants are presented. Values are mean \pm standard error of mean, of the three biological replicates. Significant differences between NFP and NTS tissues

(roots, flag leaves, grains) under a particular treatment (high $\mathrm{Fe}$ or low $\mathrm{Fe}$ ) or at a particular development stage (milky, dough, or mature) are marked with single $\left(^{*} p \leq 0.05\right)$ or double asterisks $\left({ }^{* *} p \leq 0.01\right)$, respectively. The letters $\mathrm{R}$, $\mathrm{L}$, and $\mathrm{G}$ represent root, leaf, and grain, respectively. Note the differences in scale at Y-axis for relative expression levels of tested genes. 

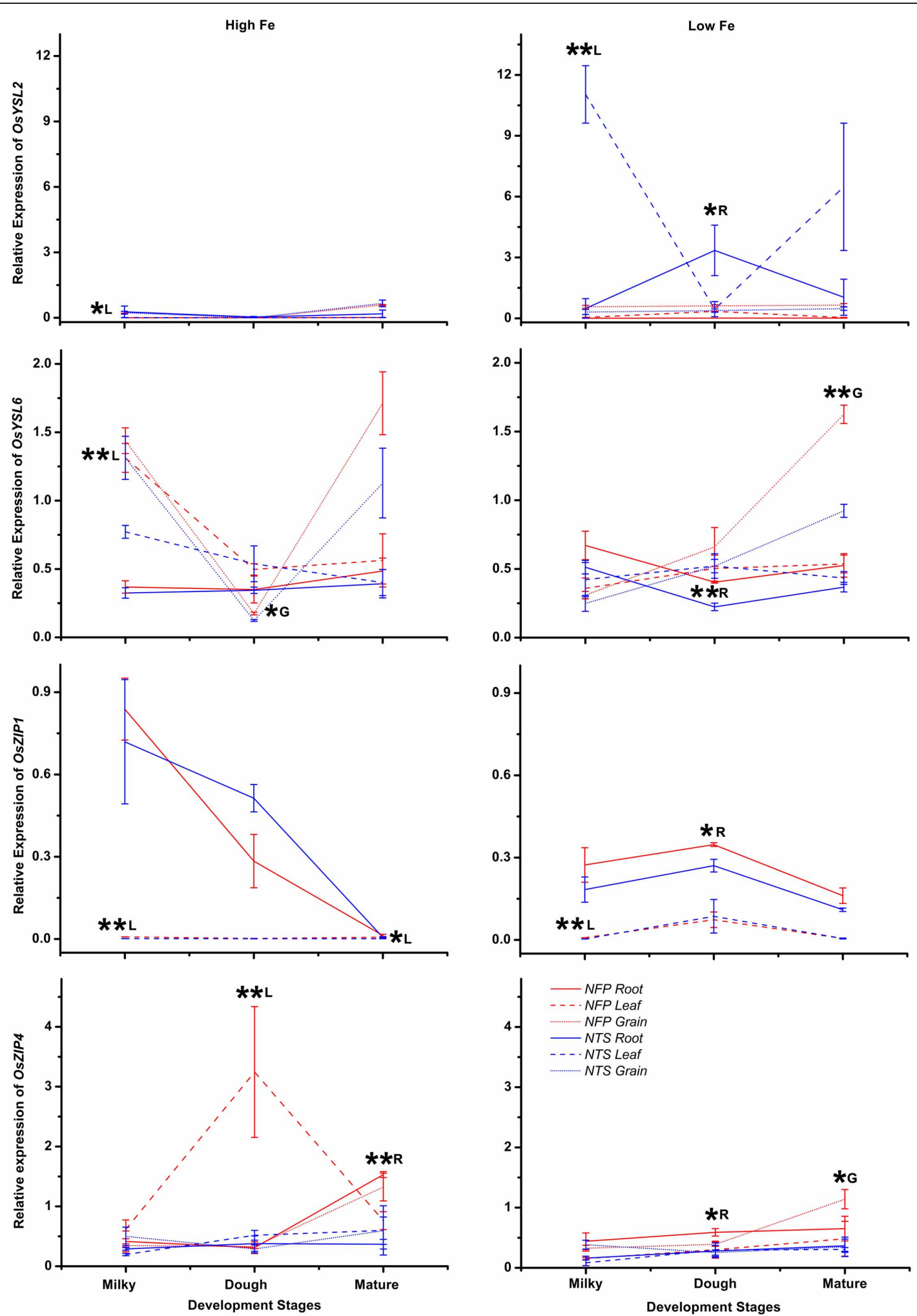

FIGURE A3 | Differences in expression levels of OsYSL2 and OsYSL6 (yellow stripe like transporters) as well as OsZIP and OsZIP4 (zinc- and iron-regulated transporters), in the roots, flag leaves, and grains of NFP vs. NTS plants are presented. Values are mean \pm standard error of mean, of the three biological replicates. Significant differences between NFP and

NTS tissues (roots, flag leaves, grains) under a particular treatment (high Fe or low Fe) or at a particular development stage (milky, dough, or mature) are marked with single $(* p \leq 0.05)$ or double asterisks $(* * p \leq 0.01)$, respectively. The letters R, L, and G represent root, leaf, and grain, respectively. Note the differences in scale at $\mathrm{Y}$-axis for relative expression levels of tested genes. 
Table A1 | Primers and probes list for real-time quantitative PCR.

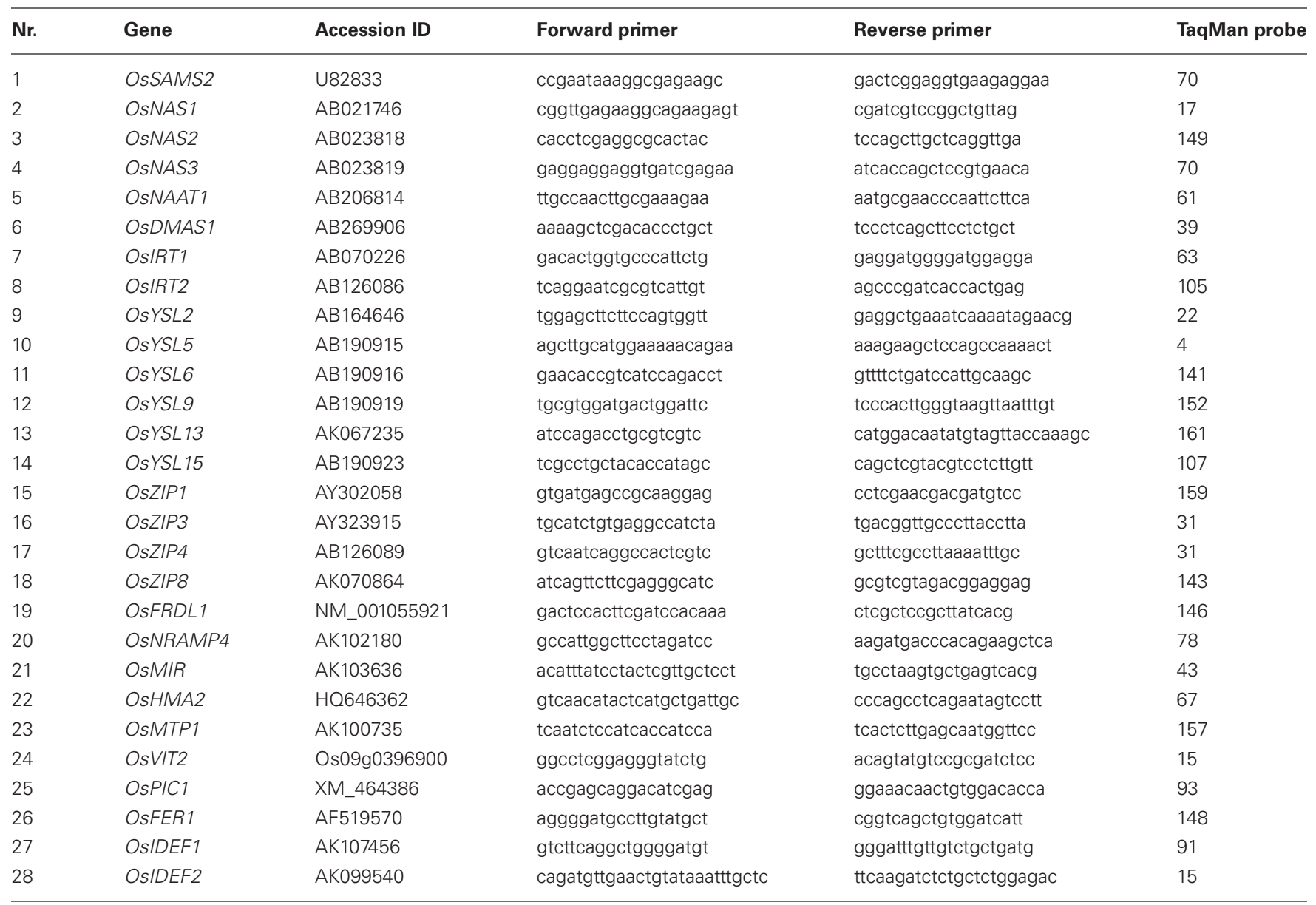

